\section{South Australian adolescent ophthalmic sun protective behaviours}

N Pakrou'1, R Casson'1, S Fung'1 , N Ferdowsi', G Lee² and D Selva ${ }^{1}$

Keywords: adolescent health; sun protection; ophthalmic protection; eye protection; ultraviolet light; sunglasses

\section{Introduction}

Appreciable evidence has accumulated to support the notion that extensive exposure to near ultraviolet (UV) radiation, and lower wavelength visible light is a risk factor for ophthalmic problems. These problems include pterygium, pinguecula, age-related macular degeneration, cataracts, ocular surface squamous neoplasia, and intraocular malignant melanoma. ${ }^{1-11}$ UV exposure may also be involved in the development of ocular melanoma and macular degeneration. ${ }^{12-14}$ Ocular sun protection practices such as wearing sunglasses (especially wrap-arounds), widebrimmed hats, and avoiding sun exposure particularly during mid-day hours have been recommended, in the attempt to minimize sun-associated ocular damage.

There have been extensive campaigns promoting bodily sun protection. Although some of these recommendations apply to ophthalmic sun protection (eg wearing wide-brimmed hats and avoiding mid-day sun), there has been a paucity of directly targeted ophthalmic sun protection campaigns. Similarly, several population studies have addressed the role of skin protection, but research specifically related to eye protection and knowledge is scant. Such information would be important in the formulation of future interventional strategies aimed at preventing excessive ophthalmic UV exposure.

As a group, the young are more susceptible to the cumulative effects of UV exposure on the skin as well as the eyes. ${ }^{11,15,16}$ Also, protective

\author{
Received: 17 March 2006 \\ Accepted in revised form: \\ 28 July 2006 \\ Published online: 20 \\ October 2006 \\ Correspondence: \\ Oplogy and Visual \\ Fax: +61882225221 \\ E-mail: nimapakrou@ \\ yahoo.co.uk
}

\begin{abstract}
Aims To study student's knowledge of the Conclusion The results of our survey suggest no significant change in knowledge and 1995 survey. We feel it is imperative that adolescents be made more aware of the damaging effects of sunlight and the benefits of eye protection. Health promotion campaigns should target the youth and consider that as a group, they are significantly influenced by the media, peers, and family attitudes.

Eye (2008) 22, 808-814; doi:10.1038/sj.eye.6702619; published online 20 October 2006
\end{abstract}


behaviours are developed during the teenage years and these behaviours will ultimately carry through into adulthood. Thus, we feel that high school students are an important target group, with respect to research into sun-related ophthalmic behaviours, and the future implementation of promotional campaigns.

Lee et $a l^{17}$ conducted a study on adolescent ocular sun protective behaviour and knowledge in Queensland Australia in 1995. In all, 652 systematically sampled Grade 10 students, aged between 13 and 17 years, were surveyed with a standardized questionnaire. Overall, this group of subjects demonstrated a moderate level of knowledge with respect to UV, sunlight, and the eyes. The knowledge of the effects of sunlight and body protection was higher than the knowledge of the effects of sunlight and eye protection. This was a well-conducted study which unfortunately only studied urban year 10 student's knowledge and attitudes. We will base our study on this, with the permission of the authors.

This study aims to elucidate the knowledge of high school students in South Australia regarding ophthalmic effects of sunlight and UV light as well as behaviours concerning eye and body protection. The preferred type of sunglasses, the frequency of use, as well as factors that determine ophthalmic sun protective behaviours are reviewed. We compare our results with the original study of 1995 by Lee G et al. ${ }^{17}$

\section{Materials and methods}

The 1995 questionnaire, taken from the study by Lee et $a l^{17}$ was used, with their permission. The survey was based on a previous instrument design and on the Health Belief Model. The following alterations were made:

(1) One class from each secondary school level (year 8-12) was surveyed in each randomly chosen school across South Australia, ensuring a good range of age groups being sampled.

(2) The surveys were administered by teachers and completed during school time, under exam-like conditions.

Ethical approval was obtained from the South Australian Department of Education and the Royal Adelaide Hospital. The study adhered to the tenants of Declaration of Helsinki and informed consent was obtained from students and parents. Schools were randomly selected in collaboration with the research department of the South Australian Department of Education to represent a range of socioeconomic areas, as well as rural and urban areas.
Students were surveyed during August-September 2004 compared to October-November 1995 by Lee et al. ${ }^{17}$ The survey was administered by teachers in each chosen school during a class session. Each response was given a score to reflect the relative appropriateness or accuracy of the response. Four sets of items were looked at, each measuring a different aspect of student knowledge. Sections included: knowledge of 'sunlight effects on the eye' - seven items (maximum score $=15$ ); 'ultraviolet light' - seven items (maximum score $=15$ ); 'eye protection'- five items (maximum score $=15$ ); 'body protection' - two items (maximum score $=14$ ). The maximum score possible was 59. Item weightings were taken from previously published studies. ${ }^{17,18}$

Several items in the survey were used to measure the relative risk of an individual's eyes to UV damage. These concerned individual phenotypic characteristics, such as eye, skin, and hair colour, and the amount of recreational outdoor exposure. The highest risk factor score of 14 was attributed to an individual with high levels of outdoor recreation, blue eyes, blond/red hair, and a fair complexion. This was based on previous studies. ${ }^{11,17,19}$ As an example of the weighting, a question asking whether ultraviolet light affects the eyes, was scores as follows: yes $=2$, not sure $=1$, no $=0$.

A number of items looked at attitudes toward the use of sunglasses. Other questions were included to examine ownership as well as the frequency of use.

The data was analysed by the analysis of variance (ANOVA), as well as the Cochran-Mantel-Haenszel methods. To determine whether awareness of the protective effects of sunglasses influences the price students are willing to pay for glasses, the Bowker's test of symmetry was used. Statistical significance was defined at the $95 \%$ level $(P<0.05)$.

\section{Results}

A total of 643 students were surveyed with 640 students (312 male and 328 female subjects) completing the survey adequately. Three surveys were not completed. Comparison of major findings between the current survey and that conducted by Lee et al is presented in Table 1. In the current study, 236 (36.9\%) students were aged 13-14 years, 227 (35.5\%) aged 15-16 years, and 177 (27.7\%) aged 17-18 years. A medium complexion was the most common skin colour reported with a one-third reporting a fair complexion. Brown was the eye colour of $233(36.4 \%)$ students and similarly blue was reported by $221(34.5 \%)$. Other eye colours were less common, with green and hazel each seen in around $14 \%$ of pupils $(n=91$ and 92, respectively). Less than 1\% $(n=5)$ had grey eyes. Looking at hair colour, about $75 \%$ reported their hair as some shade of brown. A total of 91 (14.2\%) 
Table 1 Comparison of major findings from current study to those by Lee $e t a l^{17}$

\begin{tabular}{|c|c|c|}
\hline & Pakrou et al & Lee et al \\
\hline Mean age of respondents & 15.0 years & 15.1 years \\
\hline \multicolumn{3}{|l|}{ Gender distribution } \\
\hline Male & $49 \%$ & $49 \%$ \\
\hline Female & $51 \%$ & $51 \%$ \\
\hline \multicolumn{3}{|l|}{ Recreational environment } \\
\hline $\begin{array}{l}\text { Combination of outdoor } \\
\text { and indoor }\end{array}$ & $56 \%$ & $59 \%$ \\
\hline Mainly outdoor & $36 \%$ & $33 \%$ \\
\hline Mainly indoor & $8 \%$ & $8 \%$ \\
\hline \multicolumn{3}{|l|}{ Complexion } \\
\hline Medium & $48 \%$ & $41 \%$ \\
\hline Fair & $30 \%$ & $26 \%$ \\
\hline Dark & $3.2 \%$ & $7 \%$ \\
\hline $\begin{array}{l}\text { Mean total knowledge } \\
\text { (maximum total 59) }\end{array}$ & $\begin{array}{c}37.6 \\
(\mathrm{SD}=5.1)\end{array}$ & $\begin{array}{c}37.7 \\
(\mathrm{SD}=5)\end{array}$ \\
\hline $\begin{array}{l}\text { Mean risk factor score } \\
\text { (maximum 14) }\end{array}$ & $\begin{array}{c}10 \\
\text { (range 6-13) }\end{array}$ & $\begin{array}{c}10 \\
\text { (range 4-14) }\end{array}$ \\
\hline $\begin{array}{l}\text { Aware that sunlight } \\
\text { adversely affects health } \\
\text { of eyes }\end{array}$ & $92 \%$ & $96 \%$ \\
\hline $\begin{array}{l}\text { Belief that young peoples' } \\
\text { eyes are more at risk of } \\
\text { sun damage compared } \\
\text { with that of adults }\end{array}$ & $40.6 \%$ & $47 \%$ \\
\hline $\begin{array}{l}\text { Belief that young peoples' } \\
\text { eyes are at least at equal } \\
\text { risk of sun damage } \\
\text { compared to adults }\end{array}$ & $69 \%$ & $67 \%$ \\
\hline $\begin{array}{l}\text { Aware that UV radiation is } \\
\text { harmful to the eyes }\end{array}$ & $66.4 \%$ & $82 \%$ \\
\hline $\begin{array}{l}\text { False belief that sun screen } \\
\text { applied to the face offers } \\
\text { fair to good protection to } \\
\text { the eyes }\end{array}$ & $57 \%$ & $47 \%$ \\
\hline Ownership of sunglasses & $74 \%$ & $71 \%$ \\
\hline $\begin{array}{l}\text { Percentage of students } \\
\text { 'only occasionally' or } \\
\text { 'almost never' wearing } \\
\text { sunglasses }\end{array}$ & $77 \%$ & $81 \%$ \\
\hline $\begin{array}{l}\text { Mean age of starting to } \\
\text { wear sunglasses }\end{array}$ & 9.5 years & 10.4 years \\
\hline
\end{tabular}

had blond hair, $55(8.6 \%)$ black, and only $16(2.5 \%)$ red hair.

Overall, 379 (59\%) pupils scored 10 or more on the risk factor score (maximum 14), for sun exposure. A total of 33 subjects $(5 \%)$ scored 13 or more. The median risk factor score was 10 with a range of 6-13.

\section{Knowledge scores}

Considering questions on UV effects and the eyes, the students sampled demonstrated a moderate level of knowledge, averaging $8.3(\mathrm{SD}=2.6)$ from a total of 14 . The mean total knowledge score was $37.6(\mathrm{SD}=5.1)$ from a maximum of 59. This compares to a mean score of 37.7 $(\mathrm{SD}=5)$ reported by Lee et al. ${ }^{17}$ There was a significant difference in the mean total knowledge score by age group $(F=3.37, P=0.0096)$, skin colour $(F=9.84$, $P<0.0001)$, hair colour $(F=5.88, P<0.0001)$ as well as the total risk factor score $(F=4.11, P=0.0027)$. When these variables were included together in a model, it was found that hair colour and skin colour still had a significant effect on mean total score after adjusting for age. Using post hoc comparisons for age (after adjusting for both skin and hair colour), it was found that students aged 17-18 years had a significantly higher mean total knowledge score compared to students aged 13, 14, and 15 , but not 16 years. There was a trend of increasing score with increasing age. This was different to the study by Lee et $a l^{17}$ in that the mean total knowledge score decreased with increasing age. Looking at skin colour with post hoc comparisons (adjusting for age and hair colour), students with dark skin showed a significantly lower mean total knowledge score compared to those with fair, medium or olive skin. There was however no consistent pattern with regard to hair colour and knowledge score.

Analyzing age and risk factor revealed that both these variables are still significant when adjusting for one another. Post hoc comparisons (adjusting for risk factor score) once again showed that students aged 17-18 years have a significantly higher mean total knowledge score compared to students aged 13, 14, and 15 years, but not 16 years. Looking at risk factor scores and adjusting for age, it was found that as risk factor increases, there is no consistent pattern in the mean total knowledge score.

There was no difference in the mean total knowledge score by gender $(F=0.29, P=0.59)$, eye colour $(F=1.06$, $p=0.3736)$ or main recreational activity environment $(F=1.51, P=0.2223)$.

\section{Components of knowledge questions}

\section{UV light}

Only 387 students $(60.4 \%)$ were aware that UV radiation was from the sun. A similar number $(n=400,62.5 \%)$ believed that UV is not visible, and a slightly larger number $(n=425,66.4 \%)$ were aware that UV radiation is harmful to the eyes.

\section{Eye protection}

A total of $580(91 \%)$ students believed that broadbrimmed hats provide fair to good protection for the eyes. More than half of the students $(n=366,57 \%)$, however, wrongly believed that sun screen applied to the face offers fair to good protection. Almost all students 
$(n=621,97 \%)$ agreed that sunglasses offer fair to good protection.

\section{Use of sunglasses}

The majority of students $(n=476,74 \%)$ owned a pair of sunglasses; however, 285 students (44.5\%) almost never wore their glasses. A further 208 (32.5\%) stated that they only occasionally wear their sunglasses. From the 455 students who stated the age they began wearing sunglasses, the mean age was 9.5 years $(\mathrm{SD}=3.8)$, and the median 10 years (range 1-18 years). Close to half $(n=312,49 \%)$ preferred plastic lenses, while 177 (28\%) preferred glass lenses. The majority preferred regular $(n=350,55 \%)$ as opposed to wrap-around lenses $(n=121,19 \%)$. Black lenses were the most popular $(n=189,30 \%)$, followed by mirror $(n=131,20 \%)$ and blue $(n=110,17 \%)$.

The advertising of sunglasses in various forms of media appears to have a significant affect on the frequency of wearing sunglasses. With the exception of advertising in newspapers, awareness of sunglasses advertisements on television, radio, and magazines significantly affects the frequency of wearing sunglasses (Table 2).

Students were asked how much they are willing to spend on sunglasses. They were again asked how much they are willing to spend on sunglasses knowing that they can prevent sun-related eye disorders. Our results were significant $(S=250.4358, P<0.0001)$, showing that students appear to spend at least the same amount or more on sunglasses if they consider the protective advantages of sunglasses.

\section{Personal and family attitudes}

Personal attitudes, as well as the attitudes of peers and family members regarding sunglasses, were significantly associated with the frequency of wearing sunglasses by students. Believing sunglasses to be cool significantly affected the frequency of using sunglasses $(\mathrm{CMH}=36.5000, P<0.001)$, as did believing that sunglasses protect from eye disease $(\mathrm{CMH}=10.9787$, $P=0.0041$ ), and that they should be part of the school uniform $(\mathrm{CMH}=28.5350, p<0.0001)$. Lee et $\mathrm{al}^{17}$ found similar results.

Students appear to be affected by the attitude of family members, with a significant association between frequency of wearing sunglasses and whether a student's father $(\mathrm{CMH}=18.5038, P<0.0001)$, mother $(\mathrm{CMH}=20.9291, P<00001)$, sister $(\mathrm{CMH}=27.1220$, $P<0.0001)$ or brother $(\mathrm{CMH}=12.1846, p=0.0005)$ also wear sunglasses. This was also shown by Lee et al. ${ }^{17}$ The frequency of peers wearing sunglasses also significantly influences the frequency of wearing sunglasses by students $(\mathrm{CMH}=64.0637, P<0.0001)$. Interestingly, there was no significant association between whether parents of students wanted the students to wear glasses, and their actual frequency of using sunglasses $(\mathrm{CMH}=3.4265, P=0.1803)$. A significant association was reported by Lee et al. ${ }^{17}$

\section{Discussion}

Australia is a country that receives high levels of UV radiation given its geographical location and its predominantly sunny weather. Also, it is a country in which a large proportion of the population are frequently involved in outdoor activities and hence exposed to significant levels of UV radiation. In general, over the last two decades, the number of Australians treated for sunrelated skin cancers has doubled, with a greater than one-third increase since $1995 .{ }^{20}$ There have been various media and educational campaigns aimed at informing and raising awareness of the sun's damage to the skin. There has however been much less emphasis on the damaging effects of sun exposure on the eyes. It is important that the young are made fully aware of the damaging effects of UV radiation on the eyes, as behaviours established early in life would be more likely to carry through into adult years. It is also thought that the eyes are more at risk from UV light damage in this group. ${ }^{11}$

In 1995, Lee $\mathrm{G} \mathrm{et} \mathrm{al}^{17}$ conducted a survey looking at the knowledge of sunlight effects on the eyes and protective behaviours in adolescents. The baseline characteristics of our subjects were very similar to the study by Lee et $a l,{ }^{17}$ except that in our study there was a more even distribution of students within each age group. In the original study, $88 \%$ of students were aged between 15 and 16 years.

Our study showed that more than half of the students wrongly believed that wearing sun-screen offers 'good' or 'fair' protection to the eyes, an increase of $10 \%$ percent compared to the survey conducted in 1995 (Table 1). This is of concern given that the majority of students surveyed stated spending a considerable, if not the majority of their recreational time outdoors.

The total knowledge score in our survey was almost exactly the same as the score of the students surveyed in $1995^{17}$ (Table 1). This is a little worrying as it appears that the increased efforts by various bodies over the past decade, aimed at increasing awareness in the youth, has not resulted in a significant increases in knowledge.

A survey of 2985 South Australian adolescents in 1999 found that this group was generally well aware of the association between UV radiation and skin cancer, with little variation across year levels. ${ }^{21}$ With regard to sun 
Table 2 Frequency of use of sunglasses with respect to demographic, phenotypic, and recreational exposure variables, awareness of advertisements, and mean total knowledge scores (SD)

\begin{tabular}{|c|c|c|c|c|c|}
\hline Sunglasses worn while outdoors & Almost always (47) & Usually (100) & Occasionally (208) & Almost never (285) & Significance \\
\hline Sex & & & & & $P=0.0123$ \\
\hline Male (312) & $21(6.7)$ & 49 (15.7) & $77(24.7)$ & $165(52.9)$ & $\mathrm{CMH}=6.2676$ \\
\hline Female (328) & $26(7.9)$ & $51(15.6)$ & $131(39.9)$ & $120(35.6)$ & \\
\hline Age group & & & & & $P=0.0495$ \\
\hline $13(115)$ & $4(3.5)$ & $13(11.3)$ & $42(36.5)$ & $56(48.7)$ & $\mathrm{CMH}=11.0971$ \\
\hline $14(121)$ & $2(1.7)$ & 19 (15.7) & $52(43.0)$ & 48 (38.7) & \\
\hline $15(111)$ & $7(6.3)$ & $24(21.6)$ & $28(25.2)$ & $52(46.9)$ & \\
\hline $16(116)$ & $8(6.9)$ & $18(15.5)$ & $33(28.5)$ & $57(49.1)$ & \\
\hline $17(108)$ & $16(14.8)$ & $16(14.8)$ & $28(25.9)$ & $48(44.4)$ & \\
\hline $18(69)$ & $10(14.5)$ & $10(14.5)$ & $25(36.2)$ & $24(34.8)$ & \\
\hline Skin colour & & & & & $P=0.0700$ \\
\hline Fair (192) & $17(8.9)$ & $33(17.2)$ & $55(28.7)$ & $87(45.3)$ & $\mathrm{CMH}=7.0619$ \\
\hline Medium (307) & $26(8.5)$ & $44(14.3)$ & $103(33.6)$ & $134(43.7)$ & \\
\hline Olive (120) & $4(3.3)$ & $23(19.2)$ & $43(35.8)$ & $50(41.7)$ & \\
\hline Dark (21) & $0(0.0)$ & $0(0.0)$ & $7(33.3)$ & $14(66.7)$ & \\
\hline Eye colour & & & & & $P=0.4170$ \\
\hline Blue (221) & $17(7.7)$ & $53(24.0)$ & $49(22.2)$ & $102(46.2)$ & $\mathrm{CMH}=3.9198$ \\
\hline Green (91) & $4(4.4)$ & $9(9.9)$ & $44(48.4)$ & $34(37.4)$ & \\
\hline Grey (5) & $0(0.0)$ & $0(0.0)$ & $2(40.0)$ & $3(60.0)$ & \\
\hline Brown (233) & $14(6.0)$ & $29(12.5)$ & $86(36.9)$ & $104(44.6)$ & \\
\hline Hazel (90) & $12(13.3)$ & $9(10.0)$ & $27(30.0)$ & $42(46.7)$ & \\
\hline Hair colour & & & & & $P=0.1646$ \\
\hline Blond (91) & $7(7.7)$ & 18 (19.8) & $33(36.3)$ & $33(36.3)$ & $\mathrm{CMH}=7.8511$ \\
\hline Light brown (171) & $12(7.0)$ & $31(18.1)$ & $52(30.4)$ & $76(44.4)$ & \\
\hline Medium brown (160 & $15(9.4)$ & $20(12.5)$ & $43(26.9)$ & $82(51.3)$ & \\
\hline Dark brown (147) & $12(8.2)$ & $22(15.0)$ & $54(36.7)$ & $59(40.1)$ & \\
\hline Black (55) & $0(0.0)$ & $5(9.1)$ & $22(40.0)$ & $28(50.9)$ & \\
\hline Red (16) & $1(6.3)$ & $4(25.0)$ & $4(25.0)$ & $7(43.8)$ & \\
\hline \multicolumn{6}{|c|}{ Awareness of sunglasses advertisement } \\
\hline \multicolumn{6}{|c|}{ Television } \\
\hline Yes & $34(7.2)$ & $82(17.3)$ & $164(34.5)$ & $195(41.1)$ & $P=0.0173$ \\
\hline No & $13(8.1)$ & $16(9.9)$ & $42(26.1)$ & $90(55.9)$ & $\mathrm{CMH}=5.6672$ \\
\hline \multicolumn{6}{|l|}{ Radio } \\
\hline Yes & $19(9.7)$ & 39 (19.9) & $58(29.6)$ & $80(40.8)$ & $P=0.0227$ \\
\hline No & $28(6.3)$ & $61(13.7)$ & $150(33.8)$ & $205(46.2)$ & $\mathrm{CMH}=5.1894$ \\
\hline \multicolumn{6}{|l|}{ Newspaper } \\
\hline Yes & $20(6.5)$ & $57(18.4)$ & $109(35.2)$ & $124(40.0)$ & $P=0.1491$ \\
\hline No & $27(8.2)$ & $43(13.0)$ & $99(30.0)$ & $161(48.8)$ & $\mathrm{CMH}=2.0819$ \\
\hline \multicolumn{6}{|l|}{ Magazine } \\
\hline Yes & $33(6.7)$ & $86(17.4)$ & $181(36.6)$ & $195(39.4)$ & $P=0.0083$ \\
\hline No & $14(9.8)$ & $14(9.8)$ & $27(18.9)$ & $88(61.5)$ & $\mathrm{CMH}=6.9759$ \\
\hline Main recreational environment & 27 (11.6) & $42(18.1)$ & $51(22.0)$ & $112(48.3)$ & $P=0.0834$ \\
\hline Outdoors (232) & 1 (1.9) & 9 (17.3) & 11 (21.2) & 31 (59.6) & $\mathrm{CMH}=4.9676$ \\
\hline Indoors (52) & $19(5.3)$ & $49(13.8)$ & $146(41.0)$ & $142(39.9)$ & - \\
\hline Both (356) & & & & & \\
\hline \multicolumn{6}{|l|}{ Mean knowledge } \\
\hline Score (SD) & & & & & (ANOVA) \\
\hline Sunlight & $11.6(1.8)$ & $11.0(2.2)$ & $10.6(2.2)$ & $10.2(2.4)$ & $P<0.0001, F=7.18$ \\
\hline UV & $10.0(2.2)$ & $9.5(2.1)$ & $9.2(2.1)$ & $9.2(2.2)$ & $P=0.0574, F=2.51$ \\
\hline Eye protection & $8.6(3.0)$ & $8.2(2.4)$ & $8.5(2.7)$ & $8.1(2.6)$ & $P=0.3032, F=1.22$ \\
\hline Body protection & $9.3(2.2)$ & $9.0(2.2)$ & $9.6(2.2)$ & $9.6(2.2)$ & $P=0.1540, F=1.76$ \\
\hline Total score & $39.5(4.9)$ & $37.7(5.2)$ & $37.9(5.2)$ & $37.1(5.1)$ & $P=0.0140, F=3.56$ \\
\hline Risk factor score & $10.3(1.4)$ & $10.3(1.7)$ & $9.6(1.8)$ & $9.9(1.6)$ & $P=0.0048, F=4.35$ \\
\hline
\end{tabular}

$\mathrm{CMH}=$ Cochran-Mantel-Haenszel, row percentages (\%) in brackets. 
protective behaviours, this study reported significant differences between the sexes, reporting close to $50 \%$ of male students and $30 \%$ of female students rarely or never wearing sunglasses. Similarly, our results demonstrate that males appear to wear sunglasses much less

frequently. It may be that more focus needs to be placed on educating males, as often young males may have the false belief that they are 'tougher' and less likely to be at risk. Our study showed no significant difference between male and female students when considering knowledge about sun protection and UV.

Considering the significant amount of sun exposure and related diseases in Australia, the fact that only $60.4 \%$ of students are aware that UV radiation if from the sun is cause for concern. Furthermore in our study, only twothirds of students were aware that UV is harmful to the eyes, considerably lower than that reported by Lee et al. ${ }^{17}$ This may be that there is more focus and education on sun damage in this state of Queensland, given that states predominant sunny weather year-round

We found that students aged 17-18 years had a significantly higher knowledge score compared to those aged 13 years through to 15 years of age. There was no difference between other age groups. The study by Lee et $a l^{17}$ found that knowledge score decreased with increasing age. However, only $4 \%$ of their students were aged 17 years and only $17 \%$ aged 16 years. Our study had comparable numbers in all age categories. It can be reasonable to expect that those aged 17-18 years would have a better knowledge compared to those a few years younger. Our results showed a trend of increasing knowledge score with increasing age.

Our survey showed students with dark skin to have a significantly lower mean total knowledge score compared to people with fair, medium or olive skin. It may be that these students are aware that they are at less risk of sun-related diseases, and hence are less likely to educate themselves on the topic. We were unable to demonstrate that those at highest risk (highest risk factor scores) were more likely to have a higher knowledge score.

As might be expected, advertising appears to influence the frequency of wearing sunglasses. It seems that those who have been exposed to advertisements on the television, radio, and magazines appear to be more likely to wear sunglasses. It will be useful to use the media to raise the awareness of the protective benefits of wearing sunglasses. Attempts can be made to use positive images of other youth wearing sunglasses, as both believing that sunglasses are 'cool' and the frequency of peers wearing sunglasses are significantly associated with how frequently students wear sunglasses. Efforts should also be made to emphasize that the price or colour of sunglasses does not necessarily imply better protection for the eyes, as even clear plastic alone can absorb about $95 \%$ of ultraviolet B light. ${ }^{10,22}$

Parents should be encouraged to lead by example by wearing sunglasses, as the frequency of students wearing sunglasses is significantly influenced by whether their parents wear sunglasses. As might have been predicted, students were not influenced by whether their parents insist they wear sunglasses. Our survey found that the majority of students do own a pair of sunglasses (Table 1). Despite this however, a majority wore their sunglasses either occasionally or almost never. Therefore, some of the influencing factors mentioned above should be utilized in campaigns to help increase the actual wearing of sunglasses.

The limitations of this study include the fact that only public schools were surveyed and hence students who are likely to be of higher socioeconomic status were not included. Furthermore, only school students were surveyed and hence adolescents no longer in the schooling system were also excluded. Studies in the future can aim at gathering data from both private and public sectors in order to present a sample more representative of the adolescent population.

Adolescents are a group, which although may appear resistant to various messages targeted at them by adults, are influenced greatly by what they see, and what they consider to be acceptable among their peers. Although conducted in a different state to the original survey, our study suggests that despite increasing sun-related disorders, there appears to be no apparent increase in knowledge or sun protective behaviours in the adolescent group over the past decade. Students spend a significant amount of their time in the school environment. Hence, schools should increasingly aim to emphasize the potential damage caused by exposure to sunlight and incorporate these messages in various aspects of their curriculum. They should also encourage parents to lead by example, perhaps by highlighting the issue in regular school newsletters. Informative and attractive advertisement campaigns can help to create a positive image of sunglasses and sun protective behaviours. Younger students in primary school may in fact be easier targets as they are usually less resistant to advice and instructions from adults. Once sun protective behaviours are established in earlier years, they would be much more likely to persist into adulthood.

\section{Acknowledgements}

We thank Lisa Yelland, Department of Public Health, University of Adelaide, for the kind help. 


\section{References}

1 Delcourt C, Carriere I, Ponton-Sanchez A, Lacroux A, Covacho MJ, Papoz L. Light exposure and the risk of cortical, nuclear and posterior subcapsular cataracts: the Pathologies Oculaires Liees a l'age (POLA) study. Arch Ophthalmol 2000; 118: 385-392.

2 Threlfall T, English DR. Sun exposure and pterygium of the eye: a dose-response curve. Am J Ophthalmol 1999; 128: 280-287.

3 Quandt SA, Elmore RC, Arcury TA, Norton D. Eye symptoms and use of eye protection among seasonal and migrant farm-workers. South Med J 2001; 94: 603-607.

4 Luthra R, Nemesure BB, Wu SY, Xie SH, Leske MC, Barbados Eye Studies Group. Frequency and risk factors for pterygium in the Barbados eye study. Arch Ophthalmol 2001; 119: $1827-1832$.

5 Gazzard G, Saw SM, Farook M, Koh D, Widjaja D, Chia SE et al. Pterygium in Indonesia: prevalence, severity and risk factors. Br J Ophthalmol 2002; 86: 1341-1346.

6 McCarty CA, Fu CL, Taylor HR. Epidemiology of pterygium in Victoria, Australia. Br J Ophthalmol 2000; 84: 289-292.

7 McCarty C, Taylor HR. A review of the epidemiologic evidence linking ultraviolet radiation and cataracts. Dev Ophthalmol 2002; 35: 21-31.

8 Lim R, Mitchell P, Cummings RG. Cataract associations with pinguecula and pterygium: the Blue Mountains eye study. Am J Ophthalmol 1998; 126: 717-719.

9 Panchapakesan J, Hourihan F, Mitchel P. Prevalence of pterygium and pinguecula: the Blue Mountains eye study. Aust N Z J Ophthalmol 1998; 26(Suppl): S2-S5.

10 Taylor HR. Aetiology of climatic droplet keratopathy and pterygium. Br J Ophthalmol 1980; 64: 154-163.

11 Lee GA, Williams G, Hirst LW, Green AC. Risk factors in the development of ocular surface epithelial dysplasia. Ophthalmology 1994; 101: 360-364.

12 Tucker MA, Shields JA, Hartge P, Augsburger J, Hoover RN Fraumeni Jr JF. Sunlight exposure as a risk factor for intraocular malignant melanoma. N Engl J Med 1985; 313: 789-792.

13 Cruickshanks K, Klein R, Klein BE, Nondal DM. Sunlight and the 5-year incidence of early age-related maculopathy: the Beaver Dam eye study. Arch Ophthalmol 2001; 119: 246-250.

14 Delcourt C, Carriere I, Ponton-Sanchez A, Fourrey S, Lacroux A, Papoz L, POLA Study Group. Light exposure and the risk of age-related macular degeneration: the pathologies oculaires liees a l'age (POLA) study. Arch Ophthalmol 2001; 119: 1463-1468.

15 Coombs BD, Sharples KJ, Cooke KR, Skegg DCG, Elwood JM. Variation and covariates of the number of benign nevia in adolescents. Am J Epidemiol 1992; 36: 344-345.

16 Harrison SL, Maclennan R, Apeare R, Wronski I. Sun exposure and melanocytic naevi in young Australian children. Lancet 1994; 344: 1529-1532.

17 Lee GA, Hirst LW, Sheehan M. Knowledge of sunlight effects on the eyes and protective behaviors in adolescents. Ophthal Epidemiol 1999; 6: 171-180.

18 Lee GA, Hirst LW, Sheehan M. Knowledge of sunlight effects on the eyes and protective behaviors in the general community. Ophthal Epidemiol 1994; 1: 67-84.

19 Mackenzie FD, Hirst LW, Battistutta D, Green A. Risk analysis in the development of pterygia. Ophthalmology 1992; 99: 1056-1061.

20 The Cancer Council of South Australia. Skin cancer rates continue to climb. News Release 2003; 1: 1-5.

21 Bechmann K, Conor P. SunSmart Evaluation Report No. 1. Sun protection practices among South Australian school students 1999: results of the 1999 ASSAD survey conducted in South Australia. Anti-Cancer Foundation of South Australia 2001, pp 13-26.

22 Javitt JC, Taylor HR. Absorptive lenses: the need for ocular protection. In: Meltzer DW, Isbey EK, Miller D (eds) Clinical Modules for Ophthalmologists. American Academy of Ophthalmology: San Francisco, 1991 Vol 9, module 3. 